\title{
Rap1 Signaling Prevents L-Type Calcium Channel-Dependent Neurotransmitter Release
}

\author{
Jaichandar Subramanian, ${ }^{1}$ Louis Dye ${ }^{2}$ and Alexei Morozov ${ }^{1,3,4}$ \\ ${ }^{1}$ Unit on Behavioral Genetics, Laboratory of Molecular Pathophysiology, National Institute of Mental Health and ${ }^{2}$ Microscopy and Imaging Core, National \\ Institute of Child Health and Human Development, National Institutes of Health, Bethesda, Maryland 20892, ${ }^{3}$ Virginia Tech Carilion Research Institute, \\ Roanoke, Virginia 24016, and ${ }^{4}$ School of Biomedical Engineering and Sciences, Virginia Polytechnic Institute and State University, Blacksburg, Virginia \\ 24061
}

The small GTPase Rap1 contributes to fear learning and cortico-amygdala plasticity by inhibiting glutamate release from cortical neurons, but mechanisms of this inhibition remain unknown. Conversely, L-type calcium channels (LTCCs) become involved in glutamate release after fear learning and LTP induction. Here, we show that Rap1 deletion in mouse primary cortical neurons increases synaptic vesicle exocytosis without altering endocytosis or vesicle pool size in an LTCC-dependent manner. We identify Erk1/2 as the downstream effector of Rap1 and show that its inhibition increases plasma membrane expression of LTCCs near presynaptic terminals. We propose that the Rap1 signaling enables plasticity and fear learning by regulating LTCCs at cortico-amygdala synapses.

\section{Introduction}

The small GTPase Rap1 regulates synaptic plasticity, learning, and memory by integrating and relaying information about changes in neuronal depolarization or the presence of growth factors. These changes cause protein kinase A, GTP exchange factors (GEFs), or GTPase activating proteins (GAPs) to modify Rap1 activity, which regulates various downstream effectors (Gloerich and Bos, 2011). The role of Rap1 signaling in postsynaptic plasticity is well studied. RAP-GEFs and RAP-GAPs shape spine remodeling (Xie et al., 2005; Spilker and Kreutz, 2010; Lee et al., 2011b). Rap1 acting through p38-mitogen-activated protein (MAP) kinase facilitates AMPA receptor endocytosis (Stornetta and Zhu, 2011). In contrast, the presynaptic role of Rap1 is unclear. We showed recently that Rap1 inhibits basal neurotransmitter release in the cortical input to the lateral amygdala and enables cortico-amygdala plasticity and fear learning (Pan et al., 2008). Here, using mouse primary cortical neurons, we have identified Erk1/2 signaling and L-type calcium channels (LTCCs) as the downstream Rapl effectors that mediate suppression of neurotransmitter release, which is a potential mechanism for Rap1 involvement in corticoamygdala plasticity and fear learning.

\footnotetext{
Received Nov. 30, 2011; revised Feb. 7, 2013; accepted March 11, 2013.

Author contributions: J.S., L.D., and A.M. designed research; J.S. and L.D. performed research; J.S. and L.D. analyzed data; J.S., L.D., and A.M. wrote the paper.

This work was supported by National Institute of Mental Health (NIMH) Intramural Research Program. We thank Dr. Vincent Schram, Lynne Holtzclaw, and Dr. James T. Russell [Microscopy and Imaging Core, National Institute of Child Health and Human Development, National Institutes of Health (NIH)] and Dr. Carolyn Smith at the Light Imaging Facility (National Institute of Neurological Disorders and Stroke, NIH). We also thank Tsegaye Lemma (NIMH, NIH) for help with animal maintenance.

Correspondence should be addressed to either of the following: Alexei Morozov, Virginia Tech Carilion Research Institute, 2 Riverside Circle, Roanoke, VA 24016, E-mail: alexeim@vtc.vt.edu; or Jaichandar Subramanian, The Picower Institute for Learning and Memory, Massachusetts Institute of Technology, 43 Vassar Street, Cambridge, MA 02139, E-mail: jaichandar@gmail.com.

DOI:10.1523/JNEUROSCI.5963-11.2013

Copyright $\odot 2013$ the authors $\quad 0270-6474 / 13 / 337245-08 \$ 15.00 / 0$
}

\section{Materials and Methods}

Chemicals were from Sigma or Invitrogen. U0126 [1,4-diamino-2,3dicyano-1,4-bis (o-aminophenylmercapto)butadiene] and bafilomycin were from EMD Chemicals.

Cell culture, transfection, and viral infection. Dissociated cells from cortices of E16 embryonic mice brain from both sexes were plated on glass coverslips and transfected on DIV 5 (synaptophluorin experiments) or DIV 11, as described previously (Subramanian and Morozov, 2011). The estimated cotransfection efficiency was $100 \%$ (data not shown), yet lower efficiency would only underestimate the significance of the results. Viral infection was performed on DIV 5.

DNA constructs and lentivirus. The following constructs were obtained as gifts; synaptophluorin (Gero Miesenbock, University of Oxford, Oxford, UK), B-Raf wild type (WT) and M483-B-Raf mutant (Deborah Morrison, National Cancer Institute, Fredrick, MD), and TN-XXL (Oliver Griesbeck, Max Planck Institute of Neurobiology, Martinsried, Germany). Rap1B-pBK-CMV phagemid for expressing Rap1B was isolated from a mouse hippocampal cDNA library, and the N17-Rap1B-pBK-CMV was derived from Rap1B-pBK-CMV. The lentivirus transfer vector SIN-W-PGK-Cre was made by cloning a Cre DNA fragment between BamHI and XhoI sites of SIN-W-PGK transfer vector. Lentivirus was concentrated to the titer of $5 \times 10^{8}$ transduction units per milliliter.

Live imaging and analyses. Imaging was performed using Carl Zeiss Axiovert 200M microscopes coupled to a spinning-disk confocal scan head Ultraview RS (PerkinElmer Life and Analytical Sciences). Neurons were imaged at DIV 13-DIV 15 as described previously (Subramanian and Morozov, 2011). Electrical stimulation was delivered through platinum electrodes $(10 \mathrm{~V}, 1 \mathrm{~ms}$ pulses at $10 \mathrm{~Hz}) . \mathrm{NH}_{4} \mathrm{Cl}(50 \mathrm{~mm})$ and sucrose $(500 \mathrm{~mm})$ were delivered through perfusion. Bouton fluorescence was obtained from $10 \times 10$ pixels ROIs drawn over randomly selected boutons from one cell (Subramanian and Morozov, 2011). For synaptophluorin experiments, the change in fluorescence $(\mathrm{dF})$ at a given time point was obtained by subtracting baseline fluorescence (average fluorescence for the five time points before stimulation) from the fluorescence at that time point. For bafilomycin experiments, $\mathrm{dF}$ was normalized to the average $\mathrm{dF}$ of the last five time points during stimulation in the presence of bafilomycin. 
Forster resonance energy transfer (FRET) ratio dR/R0 was calculated as $((\mathrm{R}-\mathrm{R} 0) / \mathrm{R} 0) \times 100$, where $\mathrm{R}$ is the ratio of cyan fluorescent protein (CFP) to citrine fluorescence, $\mathrm{R} 0$ is the average $\mathrm{R}$ from five time points before stimulation, and $\mathrm{dR}=\mathrm{R}-\mathrm{R} 0$.

Boutons that did not respond to stimulation or did not show fluorescence decay after stimulation was stopped were eliminated from analysis. $\mathrm{dF}$ in bafilomycin and nitrendipine (NIT) experiments is not directly comparable with $\mathrm{dF}$ in other experiments because they were performed using different setups. $\mathrm{dF}$ and $\mathrm{dR} / \mathrm{R} 0$ of a single cell were means from $7-10$ boutons. For synaptophluorin experiments, electrical stimulation lasted $18 \mathrm{~s}$, and the dFs at $6 \mathrm{~s}$ of stimulation were compared (unless mentioned otherwise). Statistical significance was determined by Student's $t$ test; repeated-measures ANOVA or one-way ANOVA followed by Dunnett's multiple comparison post hoc test using values obtained from individual cells.

Immunofluorescence. Cells were fixed with $4 \%$ PFA plus $4 \%$ sucrose and permeabilized with $0.1 \%$ Triton X-100. After blocking with $10 \%$ donkey serum (Jackson ImmunoResearch), cells were incubated with primary antibody overnight at $4^{\circ} \mathrm{C}$ and secondary antibody for $1 \mathrm{~h}$ at room temperature (RT). The primary antibodies were chicken anti-GFP (1:1000; Millipore); rabbit anti-Cre (1:1000; a gift from Christoph Kellendonk, Columbia University, New York, NY); mouse anti-phosphop42,44 MAP kinase and rabbit anti-p42,44 MAP kinase (Cell Signaling Technology); mouse anti-MAP2 (1:200, Sigma); and rabbit-anti Cav1.2 (1:50; Millipore). Secondary antibodies were Cy3-conjugated antimouse, Cy5-conjugated anti-rabbit, or DyLight 488 conjugated antichicken antibodies (1:100; Jackson ImmunoResearch). Cells were imaged using a Carl Zeiss LSM 510 microscope with $63 \times$ Plan Apochromat (1.4 numerical aperture) objective.

The phospho-Erk1/2 to total Erk1/2 ratio from immunofluorescence signals was normalized to the average ratio obtained with WT cells treated with U0126 (MEK inhibitor). To enhance the immunofluorescence signal of phospho-Erk1/2, we treated the cells with $\mathrm{KCl}$ before fixation.

Western blot and genomic PCR. Cells were lysed and centrifuged for 10 $\min$ at $12,000 \times g$ at $4^{\circ} \mathrm{C}$ (Subramanian and Morozov, 2011). The supernatant was used for Western blot. The polyvinylidene difluoride membrane (Millipore) was blocked with 1\% BSA (for Rap1 detection) or 5\% dry milk (for Erk1/2 detection) for $1 \mathrm{~h}$ at RT and incubated with primary antibody (mouse anti-Rap 1, 1:250, BD Biosciences; or rabbit anti-p42,44 MAP kinase antibody, 1:1000, Cell Signaling Technology) at $4^{\circ} \mathrm{C}$ overnight. The membrane was incubated at RT for $1 \mathrm{~h}$ with HRP-conjugated anti-rabbit or anti-mouse antibody (Jackson ImmunoResearch). The protein bands were visualized using LumiGLO reagent (Cell Signaling Technology). The pellet was used for genomic PCR as described previously (Pan et al., 2008).

Electron microscopy. Cortical neurons cultured on Aclar embedding film (Ted Pella) were treated with DMSO or U0126 for 20 min and fixed with $4 \%$ paraformaldehyde and $0.1 \%$ glutaraldehyde in PBS. After permeabilization with $0.1 \%$ Triton X-100 for 5 min at RT, cells were incubated with anti-Cav1.2 antibody (1:50; Millipore; omitted for negative control) and normal goat serum overnight at $4^{\circ} \mathrm{C}$. After washing, the cells were incubated in anti-rabbit nanogold secondary antibody (1:100; Nanoprobes) overnight at $4^{\circ} \mathrm{C}$. After silver enhancement (HQ silver enhancement kit; Nanoprobes), cells were postfixed with $0.2 \%$ osmium tetroxide for $1 \mathrm{~h}$ at RT and treated with $0.25 \%$ uranyl acetate for $1 \mathrm{~h}$ at $4^{\circ} \mathrm{C}$. After dehydration, cells were embedded in $100 \%$ resin, and $70-\mathrm{nm}-$ thick sections were prepared on a Reichert-Jung Ultracut-E ultramicrotome and placed on LuxFilm grids with a $2.0 \mathrm{~mm}$ open area and a 30 $\mathrm{nm}$ support film (Ted Pella). The grids were post-stained with uranyl acetate and lead citrate. Axons containing synaptic vesicles (SVs) and positive for immunogold labeling were imaged in a Jeol 1400 transmission electron microscope operating at $80 \mathrm{kV}$.

\section{Results}

Rap1 deletion in primary neurons increases exocytosis without altering endocytosis or vesicle pool size

We first confirmed, by using lentiviral expression, that Cre recombinase in neurons with double-floxed rapla and rap $1 b$ alleles
(Pan et al., 2008) effectively excises floxed regions in both genes and eliminates Rap1 protein (Fig. 1A). We then compared the synaptophluorin response $\mathrm{dF}$, a measure of exocytosis (Miesenböck et al., 1998), in axons of neurons chemically transfected with a construct expressing synaptophluorin (WT) or constructs expressing synaptophluorin and Cre recombinase [knock-out $(\mathrm{KO})$ ] (Fig. $1 B$ ).

We induced exocytosis by $60 \mathrm{~s}$ field electrical stimulation (Fig. $1 C, D$ ). During early phase of stimulation, at $6 \mathrm{~s}, \mathrm{dF}$ in $\mathrm{KO}$ was $95 \%$ higher than in WT terminals (Fig. 1D). However, during the later phase, at $60 \mathrm{~s}$, the $\mathrm{dF}$ was similar for both genotypes (Fig. 1D).

To confirm that the increase in $\mathrm{dF}$ in Cre-transfected cells resulted from loss of Rap1, we cotransfected constructs expressing either Rap1B or its inactive mutant N17Rap1B (N17) (Vossler et al., 1997) along with Cre and synaptophluorin. Rap1B reduced $\mathrm{dF}$ in $\mathrm{KO}$ terminals to WT levels but had no effect in WT terminals; in contrast, N17 had no effect on dF in KO axons (Fig. $1 E)$. These results show that Rap1B expression rescues Rap1 KO phenotype.

Next we tested whether the increased $\mathrm{dF}$ in $\mathrm{KO}$ neurons resulted from increased exocytosis or decreased endocytosis. In the presence of bafilomycin, which blocks vesicle reacidification, $\mathrm{dF}$ is a direct measure of exocytosis. Endocytosis can be quantified as the difference between $\mathrm{dF}$ in the presence and absence of bafilomycin (Burrone et al., 2006). We first treated WT or KO neurons with DMSO for $15 \mathrm{~min}$ and measured $\mathrm{dF}$ during $30 \mathrm{~s}$ stimulation. After another $15 \mathrm{~min}$ in DMSO and $1 \mathrm{~min}$ after addition of bafilomycin, same boutons were reimaged during $90 \mathrm{~s}$ stimulation (Fig. 2A). The time constant of exocytosis determined from a single-exponential fit of the $\mathrm{dF}$ in the presence of bafilomycin was $15.2 \pm 1.3 \mathrm{~s}$ for $\mathrm{KO}$ and $27.4 \pm 2.9 \mathrm{~s}$ for WT $(p=0.009, t$ test $)$ indicating that exocytosis is faster in the absence of Rap1.

At the end of the $90 \mathrm{~s}$ stimulation, both genotypes had similar dFs (KO, 716.2 \pm 132.3; WT, 782.2 \pm 142.5 ; $p=0.75, t$ test $)$, suggesting that total recycling pool is similar between genotypes. The level of endocytosis was not significantly different between the two genotypes at all time points (Fig. 2A,B).

We next looked at the vesicle pool size, which may influence exocytosis rate. To test the total vesicle pool, we measured increase in fluorescence caused by $\mathrm{NH}_{4} \mathrm{Cl}$, which reversibly raises the internal $\mathrm{pH}$ and enables synaptophluorin fluorescence in all SVs (Sankaranarayanan et al., 2000). The increase in fluorescence did not differ between WT and KO terminals (Fig. 2C). The readily releasable pool (RRP) exocytoses by hyperosmotic shock with sucrose (Rosenmund and Stevens, 1996), which produced the same fluorescence increase in both genotypes (Fig. 2D). These results indicate that sizes of total and RRP are not altered by Rap 1 deletion.

\section{Rap1-B-Raf-Erk1/2 pathway suppresses exocytosis}

In neurons, Rap 1 activates Erk1/2 through B-Raf and MEK (Fig. 3A) (York et al., 1998). We recently found that Erk1/2 inhibition by U0126 enhances SV exocytosis (Subramanian and Morozov, 2011). Because both Rap1 deletion and Erk1/2 inhibition enhanced exocytosis, we tested whether these proteins were acting through the same pathway. We first confirmed that interference with Rap1 or B-Raf function reduces Erk1/2 phosphorylation. We quantified phospho-Erk1/2 in KCl-induced neurons expressing Cre (KO neurons) or a dominant-negative B-Raf-K483M (Wan et al., 2004) by immunofluorescence. When compared with WT cells, the phospho-Erk1/2 to total Erk1/2 fluorescence 
A

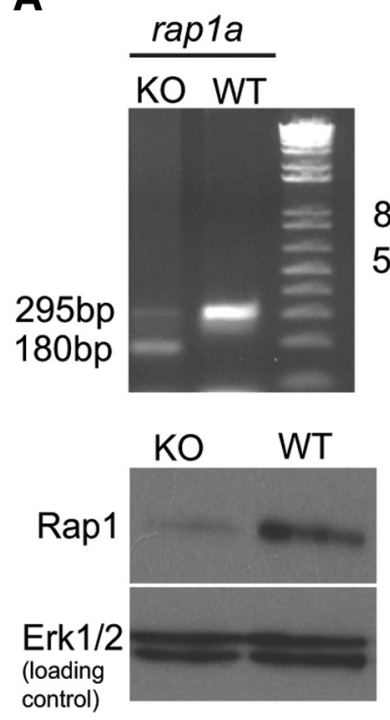

B

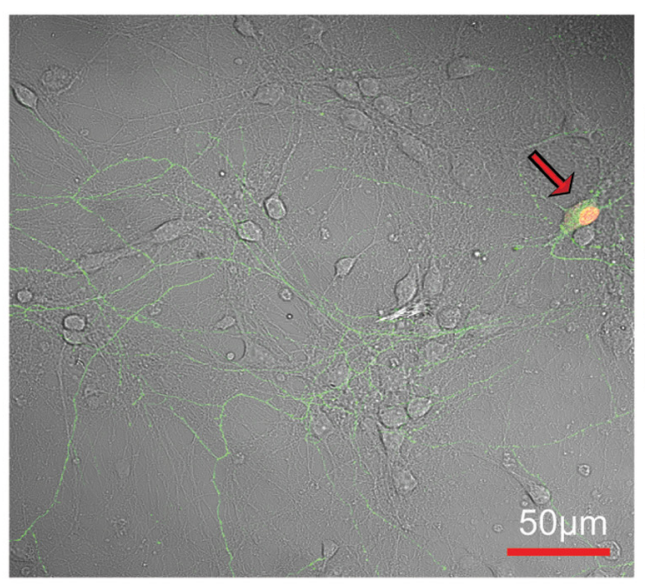

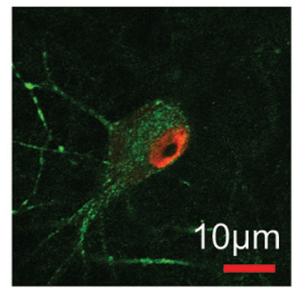

C Before stimulation End of $60 \mathrm{~s}$ stimulation
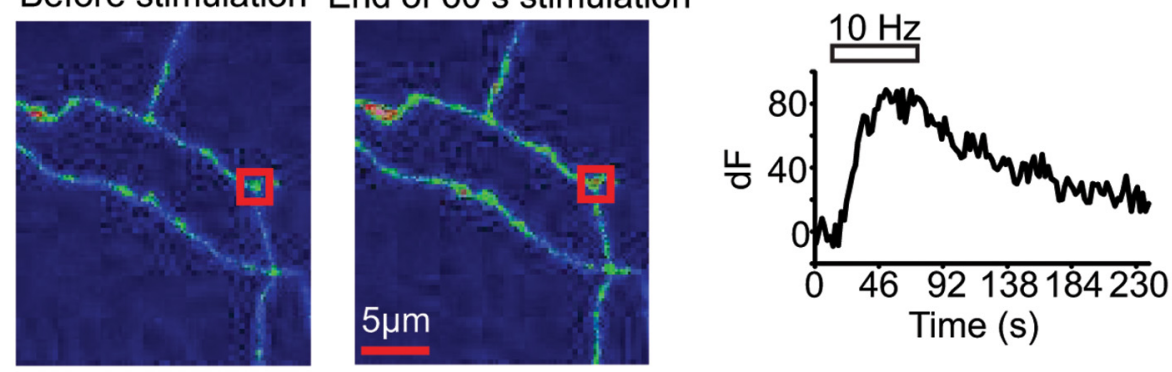

D
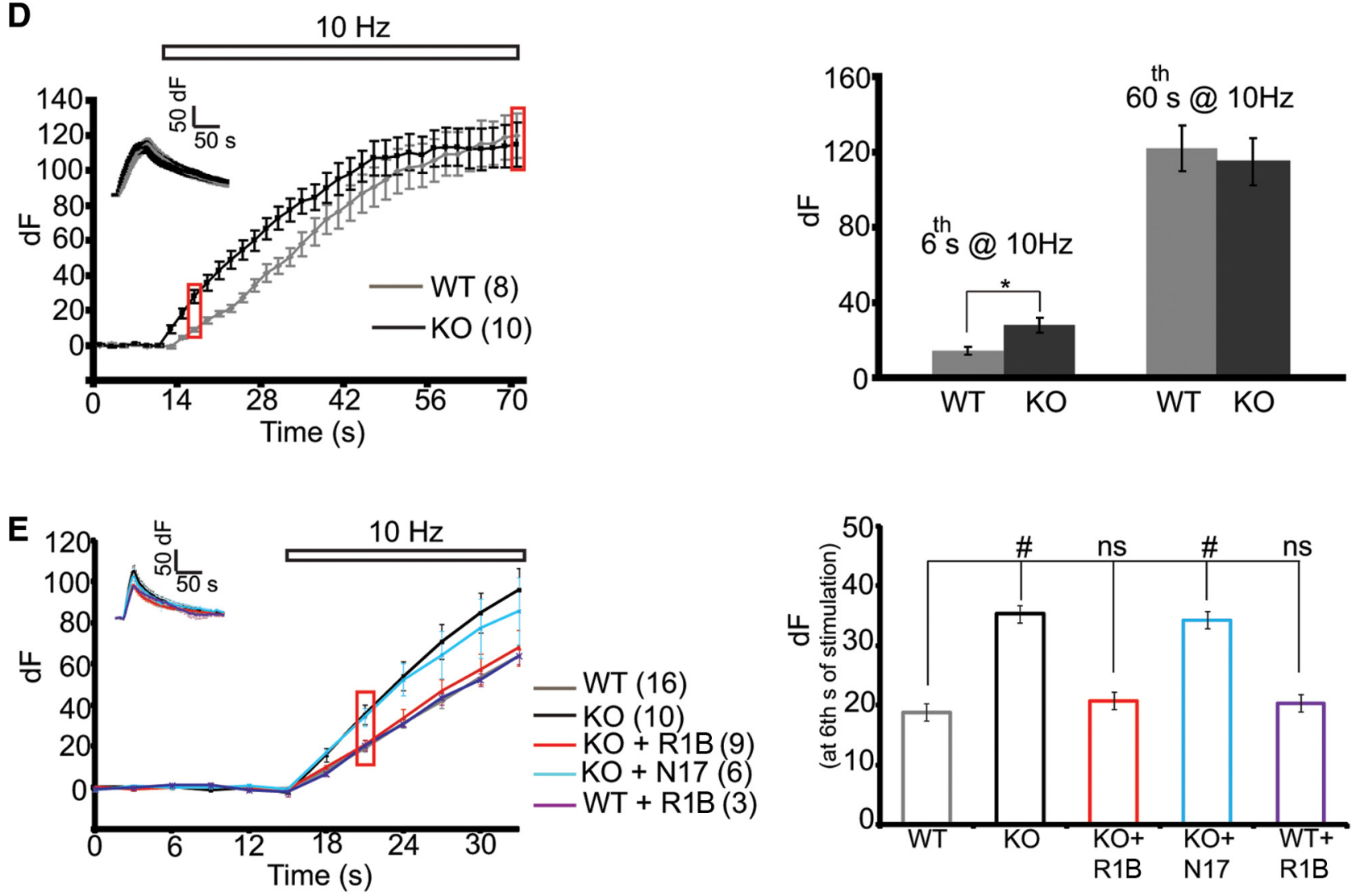
ratio was lower in both $\mathrm{KO}$ and $\mathrm{B}-\mathrm{Raf}-\mathrm{K} 483 \mathrm{M}$ cells but not in WT B-Raf construct transfected cells (Fig. $3 B, C$ ).

If Rap 1 and Erk1/2 acted through the same pathway, the Rap1 deletion would occlude the effect of Erk1/2 inhibition by U0126. At $2 \mathrm{~mm}$ external $\mathrm{Ca}^{2+}$, U0126 increased $\mathrm{dF}$ in WT cells by $65 \%$ but had no significant effect in $\mathrm{KO}$ cells (Fig. 3D), suggesting that Erk1/2 and Rap1 suppress exocytosis via a shared pathway. Alternatively, Rap1 and Erk1/2 might act independently, but exocytosis reached a ceiling in the absence of Rap 1 and masked the effect of U0126. To avoid ceiling effects, we decreased external $\mathrm{Ca}^{2+}$ concentration to $1 \mathrm{~mm}$, which reduced $\mathrm{dF}$ by half. Under these conditions, U0126 increased dF by $220 \%$ in WT terminals but only by $25 \%$ in $\mathrm{KO}$ (Fig. $3 E$ ). The effect of U0126 in KO was significantly lower than in WT $(p=0.016)$. These data suggest that inhibition of exocytosis by Erk1/2 depends primarily, but not exclusively, on the pool of Erk1/2 that is activated through the Rap1-dependent pathway. Because Rap1 activates Erk1/2 via B-Raf (Ohtsuka et al., 1996), an interference with B-Raf activity should also enhance exocytosis. Consistently, the dominantnegative B-Raf increased $\mathrm{dF}$ by $45 \%$, whereas the WT B-Raf had no effect (Fig. $3 F$ ). Together, these data indicate that interference with the Rap1-B-Raf-MEK-Erk1/2 pathway enhances exocytosis.

\section{Rap1 deletion increases calcium influx in axons through Erk1/2}

Next we examined the mechanism by which Rap1 deletion enhances SV exocytosis. Because the vesicle pool size was not altered in $\mathrm{KO}$ neurons, we tested whether calcium level was higher in axons during electrical stimulation. We used TN-XXL, a FRETbased genetic indicator of calcium (Mank and Griesbeck, 2008) (Fig. 4A). Electrical stimulation reduced CFP and increased citrine fluorescence of TN-XXL, thus increasing the FRET ratio dR/R0 (Fig. 4A). We imaged axons of neurons transfected with TN-XXL construct alone (WT) or together with Cre (KO). During $6 \mathrm{~s}$ electrical stimulation, dR/R0 increase was significantly higher in $\mathrm{KO}$ than in WT axons (KO vs WT; $A N O V A, F_{(1,7)}=3.4$; $p=0.002 ; n=10$ (WT) and 10 (KO); Fig. 4B), indicating that deletion of Rap1 increases axonal calcium influx during activity. Because inhibition of Erk1/2 also increases axonal calcium (Subramanian and Morozov, 2011), we examined effect of U0126 on $\mathrm{dR} / \mathrm{R} 0$ in KO axons. U0126 significantly increased dR/R0 in WT cells $(\mathrm{WT}+\mathrm{U} 0126$ vs WT; dR/R0 $\times$ treatment interaction; ANOVA, $F_{(1,7)}=3.1 ; p=0.006 ; n=6$ per group) but not in $\mathrm{KO}$ cells $\left(F_{(1,7)}=1.6 ; p=0.15 ; n=7\right.$ per group) (Fig. $4 C$ ). These

\footnotetext{
$\leftarrow$

Figure 1. Deletion of rap1a and rap1b genes increases synaptophluorin response. $\boldsymbol{A}$, Genomic PCR (top) and Western blot (bottom) of uninfected (WT) and lentivirus Cre-infected (KO) neurons. The 180 and 540 bp bands represent excised rap $1 a$ and rap $1 b$ alleles, respectively, and the 295 and 883 bp represent the intact alleles. $\boldsymbol{B}$, Overlay of bright-field and fluorescent images of a primary cortical neuron (shown by the arrow and enlarged image on right) cotransfected with plasmids expressing synaptophluorin (green) and Cre (red; Cre immunostaining). C, Pseudo-color image of axons expressing synaptophluorin. $\mathrm{dF}$ can be seen as a shift toward warmer colors. Red squares indicate an ROl, whose dF is shown (right). D, Left, dF before and during $60 \mathrm{~s}$ stimulation. Inset includes fluorescence decay after stimulation. Data points are separated by $2 \mathrm{~s}$. Right, Average $\mathrm{dF}$ at the time point indicated by the red boxes in the time diagram. $\boldsymbol{E}$, Left, dF before and during stimulation. Inset includes poststimulus decay. Data points are separated by $3 \mathrm{~s}$. Right, $\mathrm{dF}$ at $6 \mathrm{~s}$ of stimulation (red box in the time diagram). WT and $\mathrm{KO}(\boldsymbol{D}, \boldsymbol{E})$ corresponds to neurons transfected with synaptophluorin alone or cotransfected with Cre, respectively. R1B and N17 indicate WT Rap1B and GTP binding domain mutant, respectively. Horizontal bar shows time of stimulation. Number of cells is indicated in the parentheses. Data are expressed as mean \pm SEM. ${ }^{*} p \leq 0.05$ (t test); ${ }^{*} p \leq 0.05 ;$ ns, not significant compared with WT (Dunnett's multiple comparison test).
}

results suggest that Rap1 acts via Erk1/2 in suppressing axonal calcium influx.

We showed previously that, in cells pretreated with LTCC blocker NIT, U0126 does not increase FRET response (Subramanian and Morozov, 2011). Surprisingly, NIT did not reduce the elevated dR/R0 in Rap1 KO or cells that were pretreated with U0126 (data not shown; see Discussion). However, a 15 min treatment of neurons expressing synaptophluorin with NIT (Fig. $4 D$ ) reversibly reduced $\mathrm{dF}$ in KO neurons but not in WT neurons (Fig. 4D), which suggests that Rap1-Erk1/2 signaling pathway reduces neurotransmitter release by suppressing LTCCs.

To determine whether Erk1/2 signaling alters LTCC expression, we quantified the ratio between Cav1.2 and MAP2 immunofluorescence in dendritic segments (Fig. 4E) of cells treated with DMSO or U0126. We found no difference between the two conditions (DMSO, $0.59 \pm 0.004, n=60$ segments; U0126, $0.54 \pm 0.003, n=59$ segments; $p=0.2, t$ test), suggesting that the change in LTCC protein expression levels is not required for the observed phenotype.

We showed that Erk1/2 inhibition enhances surface fraction of Cav1.2 in the soma (Subramanian and Morozov, 2011). However, immunofluorescence does not detect Cav1.2 in axons, and it is unclear whether these channels are present near presynaptic terminals. To this end, we performed Cav1.2 immunoelectron microscopy of DMSO- or U0126-treated neurons. We analyzed axonal regions that contain SVs (Fig. $4 F$ ) and calculated the numeric ratio between immunogold particles on the membrane and in the cytoplasm. The ratio was 2.2-fold higher in U0126-treated than in DMSO-treated neurons (U0126, $59 \pm 9.5 \%, n=19$ profiles; DMSO, $26.2 \pm 8.9 \%, n=15$ profiles; $p=0.01)$. These results show that LTCC is present in axons and that Erk1/2 inhibition increases its surface expression.

\section{Discussion}

Here, we show that inhibition of Rap1 signaling increases LTCCdependent SV exocytosis. Active Rap1 interacts with multiple effectors, including Ral-GDS, a GEF for Ral A, and B-Raf, an activator of Erk signaling (Bos et al., 2001). Rap1 could modulate exocytosis through $\mathrm{Ral} \mathrm{A}$, which has been reported to increase the RRP of SVs (Polzin et al., 2002). However, KO neurons had a normal RRP, and Rap1 deletion in mice did not change the level of active Ral A in the cortex (data not shown). Therefore, the effect of Rap1 on neurotransmitter release is unlikely to occur via Ral A. Instead, our findings suggest that Rap1 suppresses neurotransmitter release through B-Raf-Erk signaling. This is in contrast to the Ras-Erk1/2 signaling that increases neurotransmitter release through synapsin phosphorylation (Kushner et al., 2005). Rap1 and Ras have been shown to play an antagonistic role (Stork, 2003; Lee et al., 2011a). With respect to neurotransmitter release, they do so by activating the same intermediate Erk1/2, raising an interesting possibility that different pools of Erk1/2 play opposing roles in neurotransmitter release.

Erk1/2 inhibition increases LTCC contribution to neurotransmitter release (Subramanian and Morozov, 2011). Consistent with the idea that Rap1 acts via the Erk1/2-LTCC pathway, the increased $\mathrm{dF}$ in KO was attenuated by the LTCC blocker NIT. The rescue of $\mathrm{dF}$, but not $\mathrm{dR} / \mathrm{R} 0$, in KO by NIT can be attributable to the fact that NIT binds only to the inactive conformation of the channel (Hess et al., 1984). LTCCs become inactivated through calcium/voltage-dependent mechanisms, which require strong and lasting depolarization. Thus, when applied acutely, NIT does not inhibit LTCCs during first 30 action potentials of a $100 \mathrm{~Hz}$ stimulation but inhibits it significantly 
A
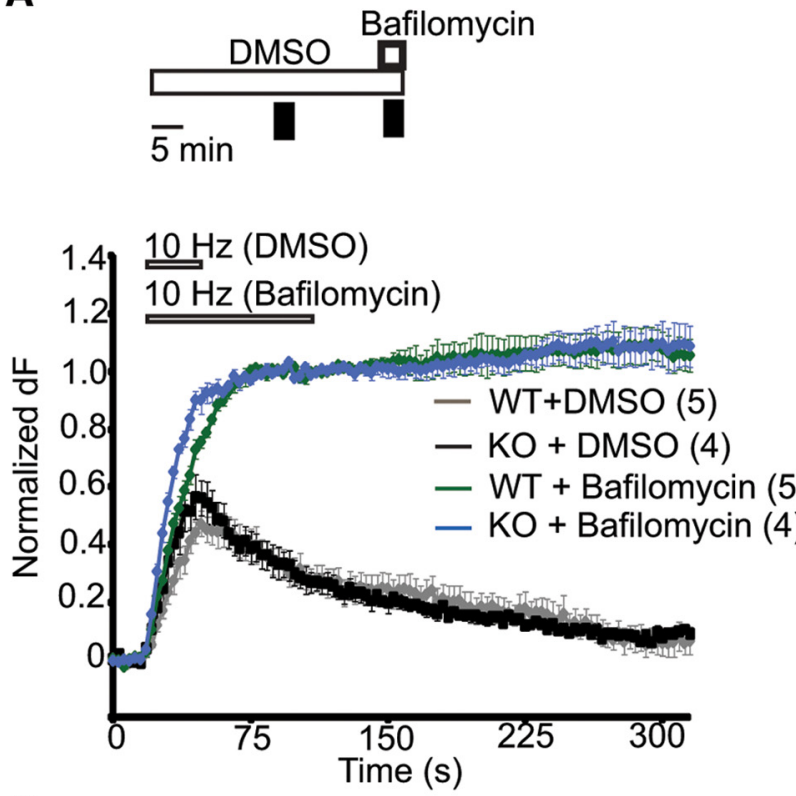

C
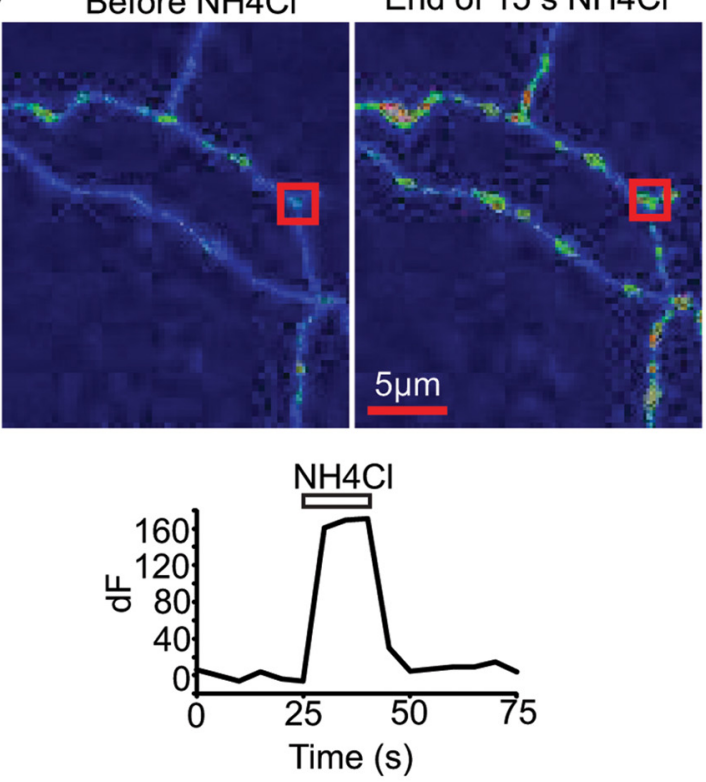

(ii)

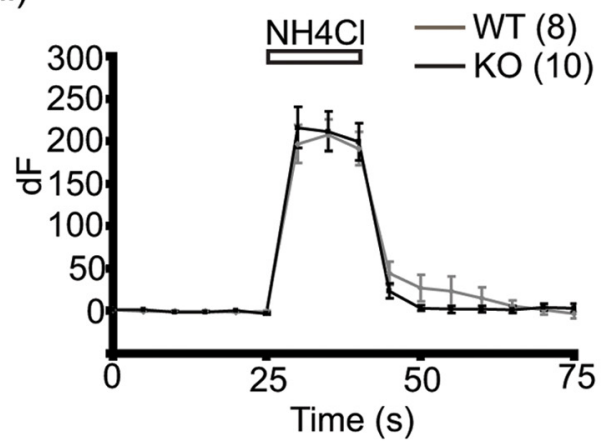

B

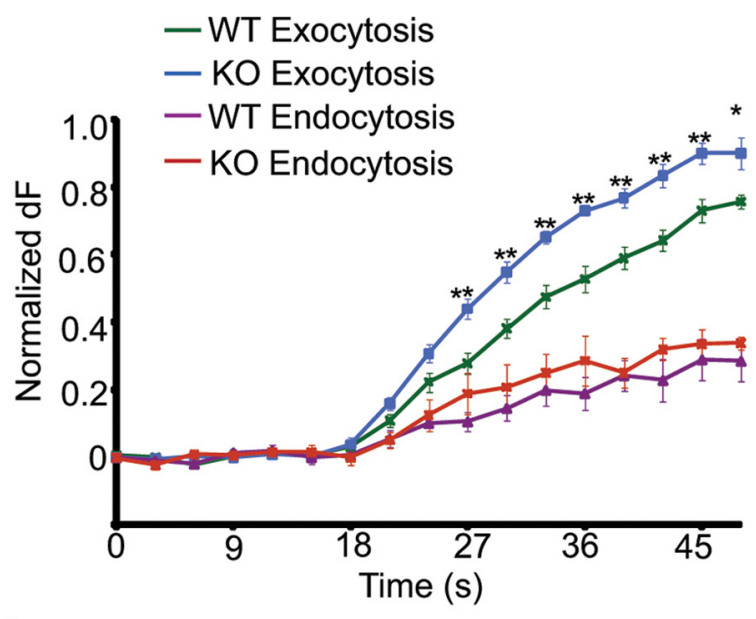

D

(i) Before Sucrose

End of $35 \mathrm{~s}$ Sucrose
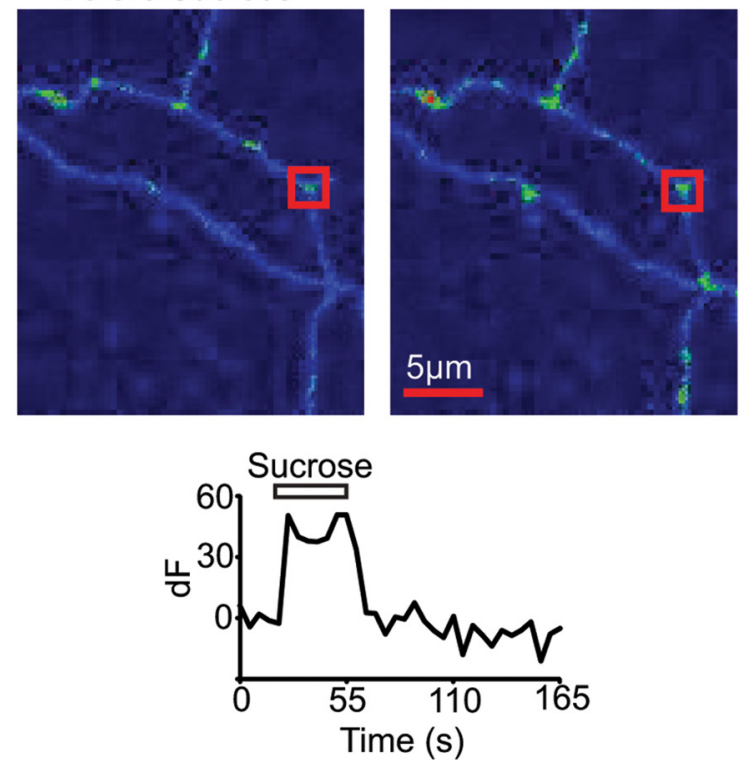

(ii)

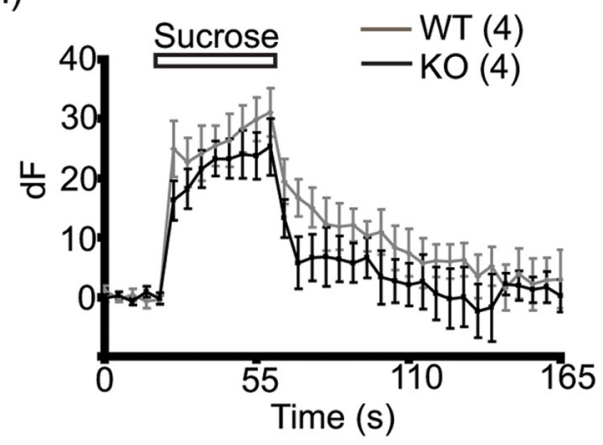

Figure 2. Rap1 deletion increases exocytosis without altering endocytosis or vesicle pool size. $\boldsymbol{A}$, Top, Experimental scheme. Black vertical bars show times of imaging. Bottom, Normalized dF (see Materials and Methods) obtained with WT and KO cells during 30 and $90 \mathrm{~s}$ stimulation in the presence of DMSO and bafilomycin, respectively. $\boldsymbol{B}$, The level of exocytosis and endocytosis determined for the first 30 s of stimulation shown in $\boldsymbol{A}$. Data points are separated by 3 s. C, $\boldsymbol{D}, \boldsymbol{i}$, Pseudo-color images of axons with synaptophluorin. A shift to warmer colors after treatment indicates $\mathrm{dF}$. Red squares indicate $\mathrm{RO}$. whose $\mathrm{dF}$ is shown (bottom). ii, Average dF dynamics. Data points are separated by $5 \mathrm{~s}$. Horizontal bar on top of graph indicates duration of stimulation. WT and KO represent cells transfected with synaptophluorin alone or cotransfected with Cre, respectively. Number of cells is indicated in parentheses. Data are represented as mean \pm SEM. ${ }^{*} p \leq 0.05$; ** $p \leq 0.01$ ( $t$ test) compared with WT exocytosis. 
A

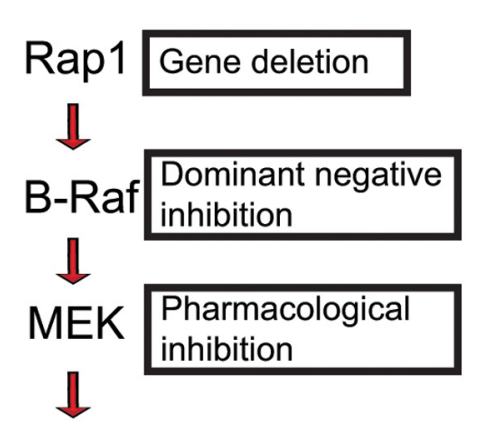

Erk1/2
B

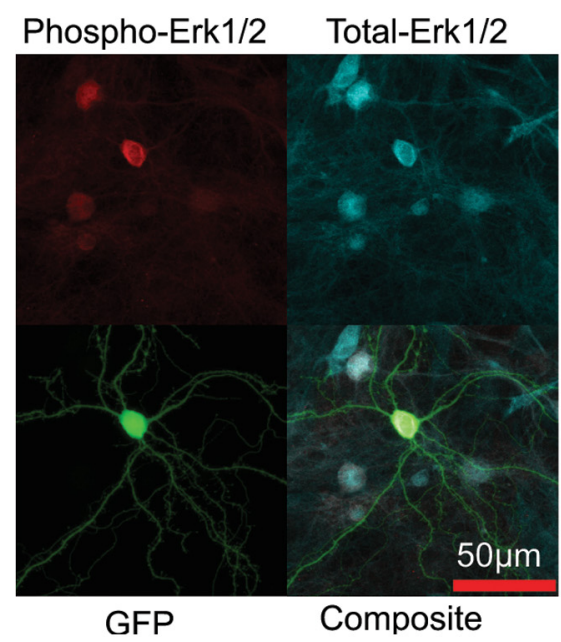

C

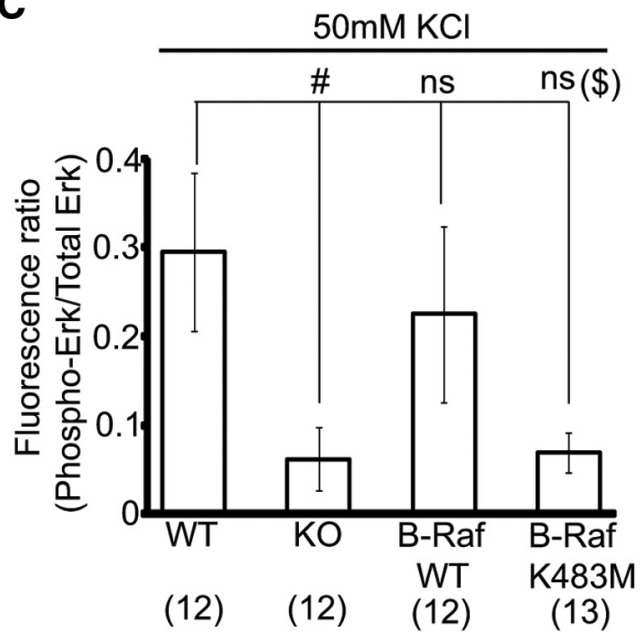

D
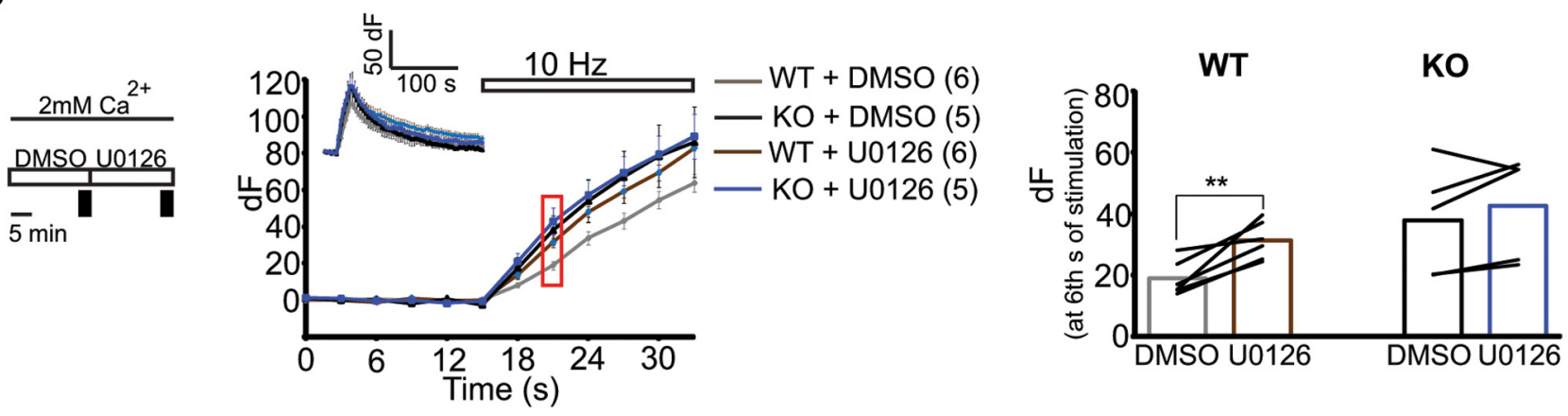

E
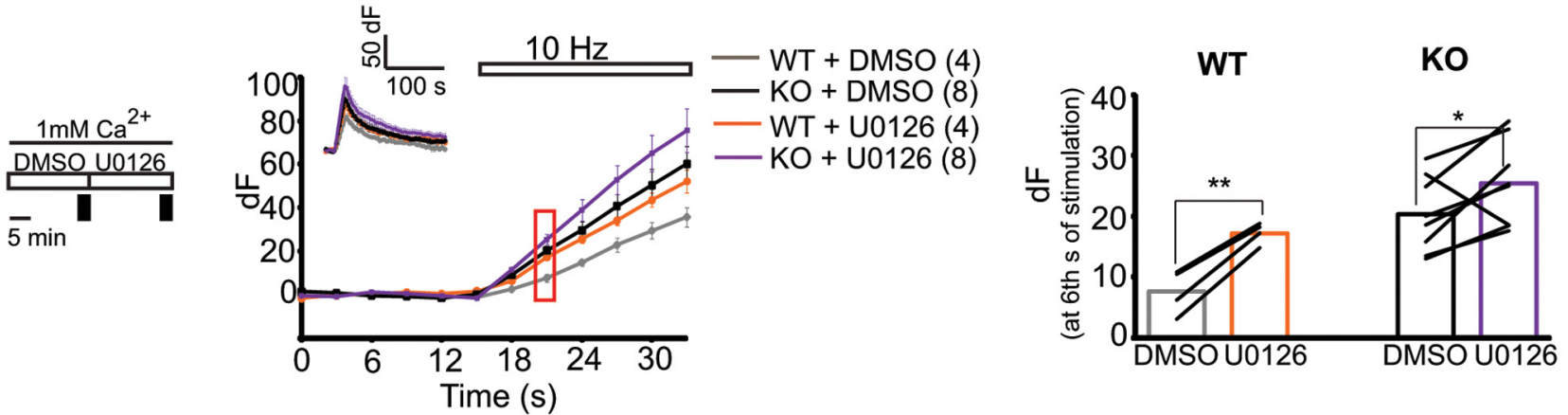

F
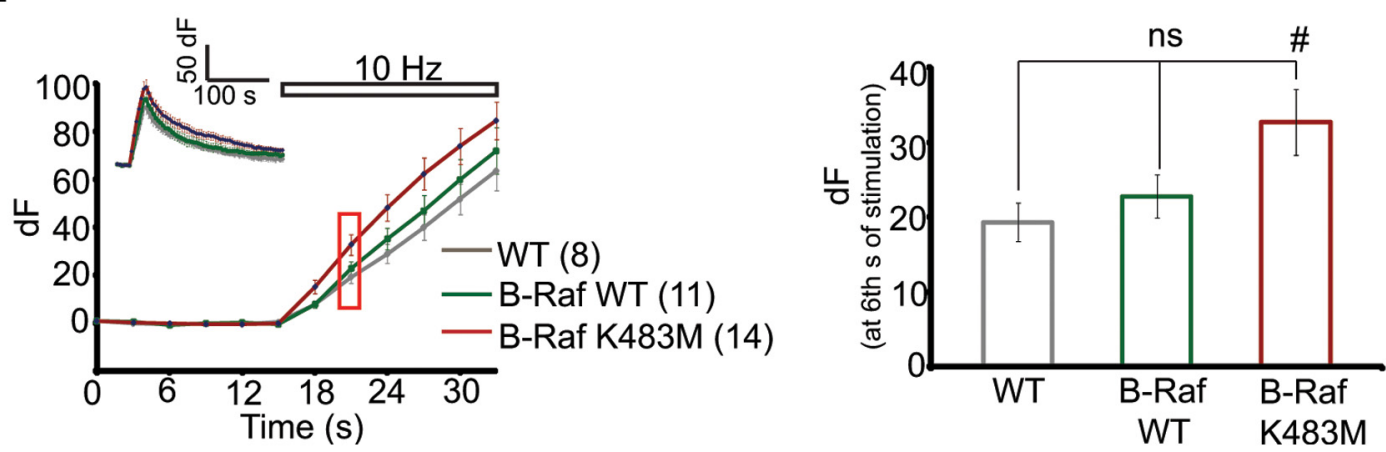

Figure 3. Rap1 regulates synaptophluorin response through Erk1/2 signaling. $\boldsymbol{A}$, Schematic representation of Rap1-Erk1/2 signaling and methods for manipulating this pathway. $\boldsymbol{B}$, A neuron transfected with a construct expressing GFP and immunostained with phospho-Erk1/2 (top left; red), total Erk1/2 (top right; blue), and GFP antibodies (bottom left; green). Overlay image is shown in bottom right. C, Normalized fluorescence ratio (see Materials and Methods) of phospho-Erk1/2 and total Erk1/2 obtained with WT (GFP transfected neurons), K0 (GFP and Cre cotransfected neurons), B-Raf WT (GFP and WT B-Raf cotransfected neurons), and B-Raf-K483M (GFP and B-Raf-K483M cotransfected neurons) after 50 mm KCI treatment. D, E, Effect of U0126 on dF in the presence of $2 \mathrm{~mm}(\boldsymbol{D})$ or $1 \mathrm{~mm}(\boldsymbol{E})$ external $\mathrm{Ca}^{2+}$. Left, Experimental schemes as in Figure 2. $\boldsymbol{F}$, Effects of B-Raf on dF. $\boldsymbol{D}-\boldsymbol{F}$, Time diagrams show dF dynamics before and during electrical stimulation. Insets include fluorescence decay after stimulation. Horizontal bars on top of graphs represent duration of stimulation. Bar graphs on the right show dF at $6 \mathrm{~s}$ of stimulation (red boxes on time diagrams). Data from individual cells are connected by black lines. Number of cells is shown in parentheses. Data are represented as mean $\pm \mathrm{SEM}$, and data points are separated by $3 \mathrm{~s} .{ }^{*} p \leq 0.05$; ${ }^{* *} p \leq 0.01$ (paired $t$ test); ${ }^{\#} p \leq 0.05 ;$ ns, not significant compared with WT (Dunnett's multiple comparison test); ${ }^{\text {} u n p a i r e d ~} t$ test (B-Raf-K483M vs WT). 
A

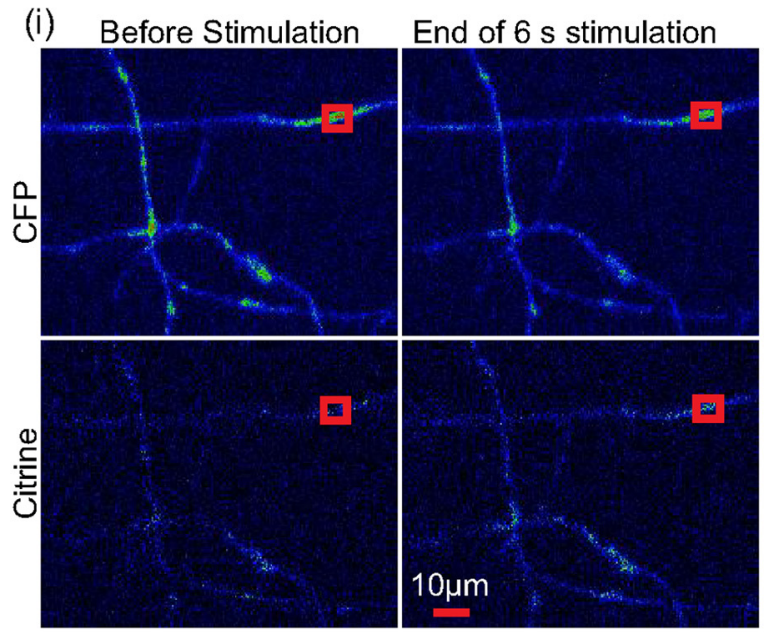

(ii)

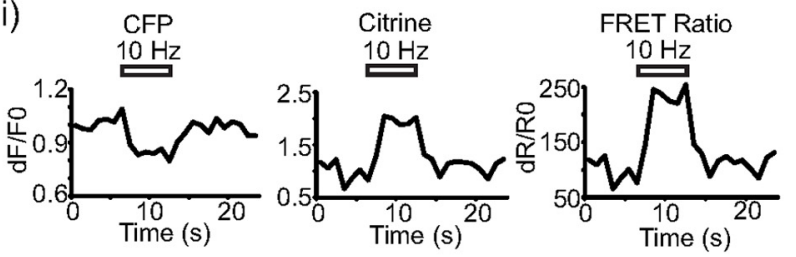

D

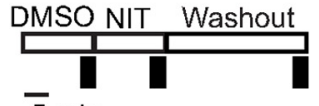

$\overline{5} \min$

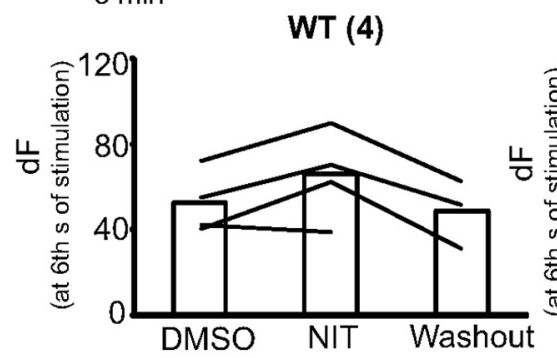

F

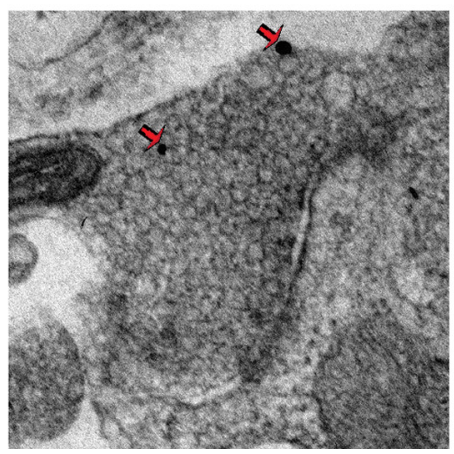

B
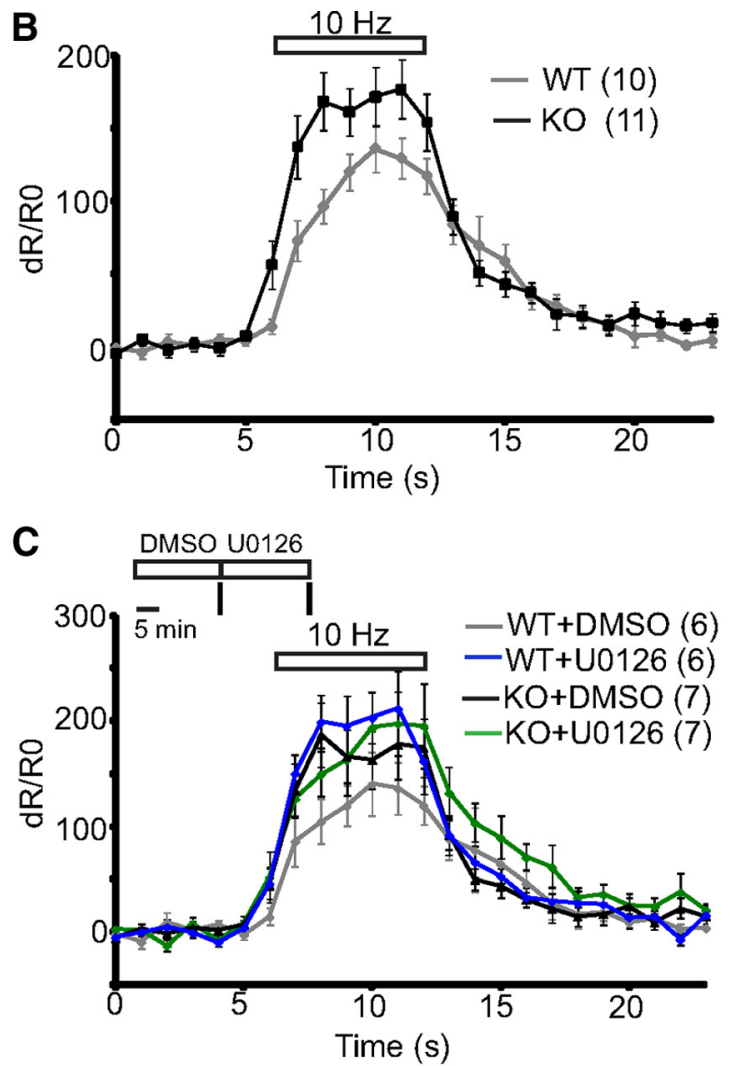

E
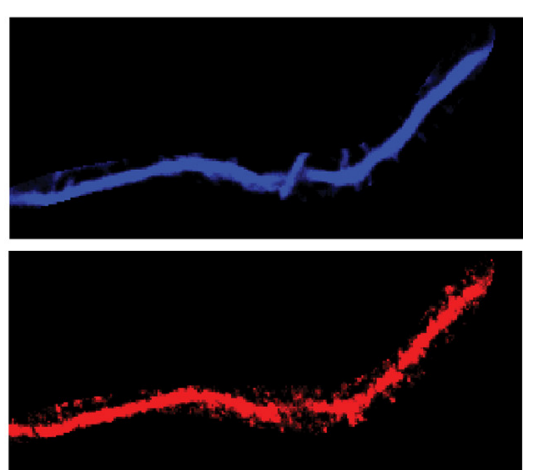

DMSO NIT Washout
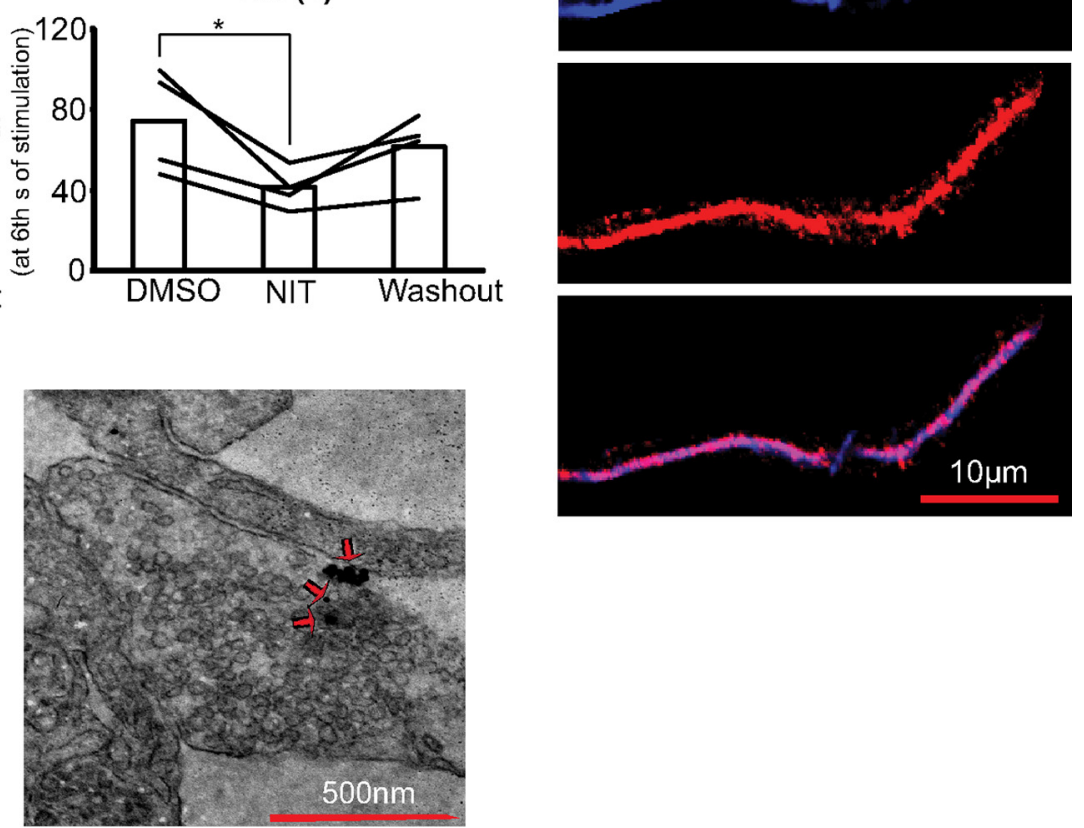

Figure 4. Rap1-Erk1/2 signaling regulates axonal calcium influx and LTCC-dependent synaptophluorin response. $A$, $i$, Pseudo-color image of axons expressing TN-XXL. CFP fluorescence decreases (shift toward cooler colors; top) and citrine fluorescence increases during stimulation (shift toward warmer colors; bottom). $i$, Response from an ROl marked by the red square. FRET ratio is defined in Materials and Methods. $B, d R / R 0$ dynamics in axons. Data points are separated by $1 \mathrm{~s}$. C, Top, Experimental scheme for testing the effect of U0126 on FRET ratio. Black vertical bars show times of imaging. Bottom, dR/RO dynamics. D, Top, Experimental scheme to test the effect of NIT on dF. Bottom, dF at $6 \mathrm{~s}$ of stimulation in WT (left) and KO (right) cells. $E$, Representative images of dendritic segments stained with antibodies to MAP2 (top) and Cav1.2 (middle). Merged image is shown at the bottom. $F$, Examples of immunoelectron microscopy images of axons with SVs. Arrows indicate immunogold labeling of Cav1.2 channels. WT represents neurons transfected with TN-XXL (B, C) or synaptophluorin (D); KO represents neurons cotransfected with Cre and either TN-XXL $(\boldsymbol{B}, \boldsymbol{C})$ or synaptophluorin (D). Number of cells is shown in parentheses. Data from individual cells are linked by a black line $(\boldsymbol{C}, \boldsymbol{D})$. Horizontal bars on top of the graphs represent duration of stimulation. ${ }^{*} p \leq 0.05$ ( $t$ test). 
after 60 action potentials (Helton et al., 2005). Similarly, in our experiments, NIT is unlikely to block LTCCs during the first 30 stimuli of even lower frequency of $10 \mathrm{~Hz}$. Because dR/R0 response saturates within 30 action potentials, it is not influenced by changes in calcium influx from NIT block of LTCCs. Conversely, synaptophluorin enables detection of NIT effect on presynaptic release after 60 stimuli because LTCCs become available for NIT blockade.

It is unclear how the presence of NIT before and during U0126 treatment was able to prevent the enhancement of dR/R0 (Subramanian and Morozov, 2011). Possibly, previous electrical stimulation in the presence of NIT was sufficient to inactivate LTCCs introduced by U0126 exposure.

Rap1-Erk signaling can promote LTCC-dependent neurotransmitter release through changes in surface expression and/or gating properties of either Cav1.2 or Cav1.3 channels. Although we did not distinguish between Cav1.2 and Cav1.3 channels, the immunoelectron microscopy of Cav1.2 channels suggests that changes in surface fraction of these channels could be a potential mechanism by which Rap1 regulates neurotransmitter release.

LTCC-dependent neurotransmitter release is enhanced after fear learning or induction of presynaptic plasticity (ShinnickGallagher et al., 2003; Fourcaudot et al., 2009). Meanwhile, the plasticity and learning deficits in Rap1 KO mice likely result from elevated LTCC-dependent neurotransmitter release. We propose that Rap1-Erk1/2 signaling enables fear learning by suppressing basal LTCC-dependent release of glutamate and thereby reducing the detection threshold of activity at cortico-amygdala synapses.

\section{References}

Bos JL, de Rooij J, Reedquist KA (2001) Rap1 signalling: adhering to new models. Nat Rev Mol Cell Biol 2:369-377. CrossRef Medline

Burrone J, Li Z, Murthy VN (2006) Studying vesicle cycling in presynaptic terminals using the genetically encoded probe synaptopHluorin. Nat Protoc 1:2970-2978. CrossRef Medline

Fourcaudot E, Gambino F, Casassus G, Poulain B, Humeau Y, Lüthi A (2009) L-type voltage-dependent $\mathrm{Ca}(2+)$ channels mediate expression of presynaptic LTP in amygdala. Nat Neurosci 12:1093-1095. CrossRef Medline

Gloerich M, Bos JL (2011) Regulating Rap small G-proteins in time and space. Trends Cell Biol 21:615-623. CrossRef Medline

Helton TD, Xu W, Lipscomber D (2005) Neuronal L-type calcium channels open quickly and are inhibited slowly. J Neurosci 25:10247-10251. CrossRef Medline

Hess P, Lansman JB, Tsien RW (1984) Different modes of Ca channel gating behaviour favoured by dihydropyridine Ca agonists and antagonists. Nature 311:538-544. CrossRef Medline

Kushner SA, Elgersma Y, Murphy GG, Jaarsma D, van Woerden GM, Hojjati MR, Cui Y, LeBoutillier JC, Marrone DF, Choi ES, De Zeeuw CI, Petit TL,
Pozzo-Miller L, Silva AJ (2005) Modulation of presynaptic plasticity and learning by the $\mathrm{H}$-ras/extracellular signal-regulated kinase/synapsin I signaling pathway. J Neurosci 25:9721-9734. CrossRef Medline

Lee KJ, Hoe HS, Pak DT (2011a) Plk2 Raps up Ras to subdue synapses. Small GTPases 2:162-166. CrossRef Medline

Lee KJ, Lee Y, Rozeboom A, Lee JY, Udagawa N, Hoe HS, Pak DT (2011b) Requirement for Plk2 in orchestrated ras and rap signaling, homeostatic structural plasticity, and memory. Neuron 69:957-973. CrossRef Medline

Mank M, Griesbeck O (2008) Genetically encoded calcium indicators. Chem Rev 108:1550-1564. CrossRef Medline

Miesenböck G, De Angelis DA, Rothman JE (1998) Visualizing secretion and synaptic transmission with $\mathrm{pH}$-sensitive green fluorescent proteins. Nature 394:192-195. CrossRef Medline

Ohtsuka T, Shimizu K, Yamamori B, Kuroda S, Takai Y (1996) Activation of brain B-Raf protein kinase by Rap1B small GTP-binding protein. J Biol Chem 271:1258-1261. CrossRef Medline

Pan BX, Vautier F, Ito W, Bolshakov VY, Morozov A (2008) Enhanced cortico-amygdala efficacy and suppressed fear in absence of Rap1. J Neurosci 28:2089-2098. CrossRef Medline

Polzin A, Shipitsin M, Goi T, Feig LA, Turner TJ (2002) Ral-GTPase influences the regulation of the readily releasable pool of synaptic vesicles. Mol Cell Biol 22:1714-1722. CrossRef Medline

Rosenmund C, Stevens CF (1996) Definition of the readily releasable pool of vesicles at hippocampal synapses. Neuron 16:1197-1207. CrossRef Medline

Sankaranarayanan S, De Angelis D, Rothman JE, Ryan TA (2000) The use of pHluorins for optical measurements of presynaptic activity. Biophys J 79:2199-2208. CrossRef Medline

Shinnick-Gallagher P, McKernan MG, Xie J, Zinebi F (2003) L-type voltage-gated calcium channels are involved in the in vivo and in vitro expression of fear conditioning. Ann N Y Acad Sci 985:135-149. CrossRef Medline

Spilker C, Kreutz MR (2010) RapGAPs in brain: multipurpose players in neuronal Rap signalling. Eur J Neurosci 32:1-9. CrossRef Medline

Stork PJ (2003) Does Rap1 deserve a bad Rap? Trends Biochem Sci 28:267275. CrossRef Medline

Stornetta RL, Zhu JJ (2011) Ras and Rap signaling in synaptic plasticity and mental disorders. Neuroscientist 17:54-78. CrossRef Medline

Subramanian J, Morozov A (2011) Erk1/2 inhibit synaptic vesicle exocytosis through L-type calcium channels. J Neurosci 31:4755-4764. CrossRef Medline

Vossler MR, Yao H, York RD, Pan MG, Rim CS, Stork PJ (1997) cAMP activates MAP kinase and Elk-1 through a B-Raf- and Rap1-dependent pathway. Cell 89:73-82. CrossRef Medline

Wan PT, Garnett MJ, Roe SM, Lee S, Niculescu-Duvaz D, Good VM, Jones CM, Marshall CJ, Springer CJ, Barford D, Marais R; Cancer Genome Project (2004) Mechanism of activation of the RAF-ERK signaling pathway by oncogenic mutations of B-RAF. Cell 116:855-867. CrossRef Medline

Xie Z, Huganir RL, Penzes P (2005) Activity-dependent dendritic spine structural plasticity is regulated by small GTPase Rap1 and its target AF-6. Neuron 48:605-618. CrossRef Medline

York RD, Yao H, Dillon T, Ellig CL, Eckert SP, McCleskey EW, Stork PJ (1998) Rap1 mediates sustained MAP kinase activation induced by nerve growth factor. Nature 392:622-626. CrossRef Medline 